\title{
The impact of pelvic floor muscle training on the quality of life of women with urinary incontinence: a systematic literature review
}

\author{
Agnieszka Radzimińska \\ Agnieszka Strączyńska \\ Magdalena Weber-Rajek \\ Hanna Styczyńska \\ Katarzyna Strojek \\ Zuzanna Piekorz \\ Department of Physiotherapy, \\ Nicolaus Copernicus University \\ Collegium Medicum in Bydgoszcz, \\ Bydgoszcz, Poland
}

This article was published in the following Dove Press journal:

Clinical Interventions in Aging

\begin{abstract}
Purpose: The purpose of this review was to assess the effectiveness of pelvic floor muscle training (PFMT) in the treatment of urinary incontinence (UI) in women, with a particular focus on the impact of this form of therapy on the patients' quality of life (QoL).

Methods: The following electronic databases were searched: PubMed, Embase, and Cochrane Library (articles only in English, 1990-2017). Search terms were as follows: urinary incontinence, pelvic floor muscle training, pelvic floor exercises, quality of life. Systematic review methods were based on the PRISMA (Preferred Reporting Items for Systematic Reviews and Meta-Analyses) statement.

Results: The assessment of the impact of PFMT on the QoL of women with UI was conducted among 2,394 women in 24 selected studies. After the end of treatment, the majority of patients in the experimental groups noted a statistically significant improvement in QoL.

Conclusion: The results of this literature review demonstrate that PFMT is an effective treatment for UI in women. PFMT significantly improves the QoL of women with UI, which is an important determinant of their physical, mental, and social functioning.
\end{abstract}

Keywords: urinary incontinence, pelvic floor muscle training, quality of life

\section{Introduction}

The frequency of urinary incontinence (UI) is difficult to determine, and there is no unequivocal data indicating the percentage of people suffering from this disorder. According to the data adopted during the 6th International Consultation on Incontinence (ICI), the problem of UI in the whole population ranges from $4 \%$ to $8 \% .{ }^{1}$ It is worth noting that the number of people affected by this condition in the entire world population has been steadily growing over the last decade. In 2008, 346 million people suffered from UI, while in 2013, 383 million. At the same time, it was estimated that in 2018, the number of people with UI around the world would be $\sim 420$ million 300 million women and 120 million men. ${ }^{2}$

Based on etiology and pathophysiology, UI is classified into 3 types: Stress UI (SUI), Urge UI (UUI), and Mixed UI (MUI). SUI is defined as the involuntary leakage of urine during exertion, such as coughing, sneezing, or laughing. An increase in abdominal pressure due to physical exertion puts stress on the bladder, causing urine to leak. The basic mechanisms responsible for that reaction are poor urethral support by the pelvic floor muscles and intrinsic sphincter deficiency. Many factors predisposing to SUI are identified in the related literature, and among others, these are as follows, numerous births, previous operations, generalized weakening of connective
Correspondence: Agnieszka Strączyńska Department of Physiotherapy, Nicolaus Copernicus University Collegium Medicum in Bydgoszcz, Techników Street 3, Bydgoszcz 85-80I, Poland Email a.straczynska@icloud.com 
tissue, hormone deficiency, genital mutilation, or reduction of reproductive organs. ${ }^{1}$ UUI (commonly referred to as "overactive bladder") is the involuntary leakage of urine preceded by a strong and sudden urge to urinate. This incontinence is usually caused by involuntary contractions of the detrusor muscles of the bladder wall at inappropriate times. UUI may be triggered by simple everyday occurrences, such as the sound of running water, exposure to cold temperatures, or drinking cold beverages. Idiopathic etiologic factors include myogenic, neurogenic, and urethrogenic symptoms. ${ }^{2}$ MUI is a mixture of SUI and UUI. Etiologic factors for MUI are also a combination of the factors described earlier. ${ }^{3}$

In addition to the described somatic symptoms, problems related to urinary iodine excretion have a psychological impact and substantially reduce patients' quality of life (QoL). They often experience discomfort, low self-esteem, mood deterioration, and the feeling of helplessness. There is a decline in quality of their personal, social, and professional life. Due to the fear of others finding out about their unfortunate condition, patients are forced to change their lifestyle, which, in turn, has a negative impact on socializing, and contributes to alienation, social isolation, changes in sexual activity, and even depression or anxiety disorder. ${ }^{4-8}$ Therefore, while assessing the effectiveness of various treatments for UI, it is essential to take into consideration the lifestyle factor, which is defined as physical, mental, and social well-being.

Physiotherapy seems to be an important part of conservative treatment of UI. Pelvic floor muscle training (PFMT) stands out among physiotherapy methods used in the treatment of UI. The scientific basis for pelvic floor muscle rehabilitation was founded by an American gynecologist Arnold Kegel, who, in the 1950s, published results of a 15-year-old study, which covered the use of pelvic floor exercises in patients with UI. Kegel pointed out that systematic muscle activity causes muscles to lose 4 times less of their mass than compared with staying inactive, and hence it is the optimal method for improving the anatomical and functional impairment of muscles. Systematic activity of contractile fragments of impaired muscles improves their coordination. This contributes to a better urethral compression, when the intraabdominal pressure increases, during exercise. Performing pelvic floor exercises correctly, especially in sensorimotor training sessions, leads to regaining proprioception in the levator ani. ${ }^{9}$

\section{The purpose of the systematic review}

The purpose of this review was to assess the effectiveness of PFMT in the treatment of UI in perimenopausal women, with a particular focus on the impact of this form of therapy on the patients' QoL. The following databases were searched: PubMed, Embase, and Cochrane Library (articles only in English, 1990-2017). The search terms used were as follows: urinary incontinence "AND" pelvic floor muscle training "OR" pelvic floor exercises "AND"/“OR" quality of life. Systematic review methods were based on the PRISMA (Preferred Reporting Items for Systematic Reviews and Meta-Analyses) statement. ${ }^{10}$

\section{Study selections}

The first and primary criterion of the review was the use of pelvic floor exercises in the treatment of UI. Then, the studies were selected based on the impact of PFMT on the QoL of women with UI. The exclusion of studies with biofeedback, electrostimulation, surgical and medical treatments was the final criterion. Quality of the selected studies was assessed using the Jadad scale. Figure 1 shows a detailed flowchart of search and exclusion strategy.

\section{Data collection}

The following data were collected from the selected studies: author name, year of publication, participants (number and age), types of UI, treatment duration, treatment type, objectivization methods of treatment outcomes, main conclusions, and the statistical analysis used. Three authors performed independent data extraction in electronic databases (AS, PubMed; MWR, Embase; and HS, Cochrane Library). One researcher collected data and conducted the data selection process (AS). Moreover, the methodological quality of the selected studies was assessed using the Jadad scale. The Jadad score is often used to assess the methodological quality of controlled trials. Studies are scored according to the presence of 3 key methodological features of clinical trials: randomization, masking, and accountability of all patients, including withdrawals. One point is added for an affirmative answer ("yes") to each of the first 5 items, while 1 point is subtracted for an affirmative answer ("yes") from either of the last 2 items, for an overall score from 0 to $5 .{ }^{11}$ The systematic analysis includes only clinical studies with a score of 3 or higher in the Jadad scale. Two authors (AS and MWR) individually assessed the methodological quality of the collected research using the Jadad scale.

\section{Results and discussion Study characteristics}

Electronic medical databases were searched in November 2017. The search retrieved 3,680 results: a total of 464 in the Cochrane Library, 3,016 in Embase, and 208 in 


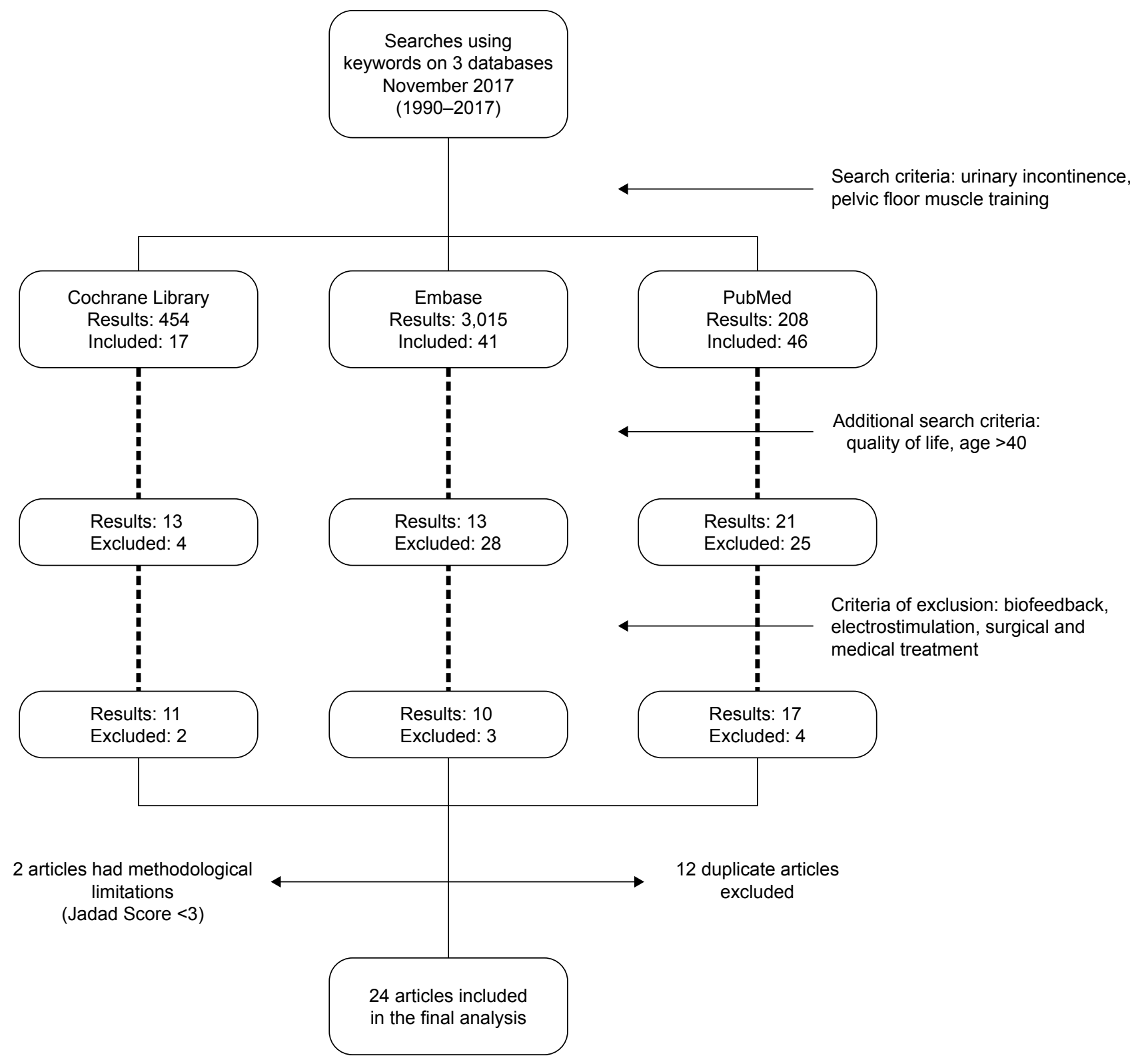

Figure I Flowchart demonstrating the process of selecting studies.

PubMed. The studies were selected using a very detailed flowchart (Figure 1). Twenty-four articles were included in the final review (Table 1). There were between 30 and 446 women (a total of 2,394 patients), aged 40-85 years old in each study group. The Jadad scale showed that 19 research findings were randomized and additionally 5 of them were blinded.

\section{Outcome measures}

The following diagnostic tests were used in the research studies: the number of UI episodes and the pad test and urinary diary. Pelvic floor muscle strength and function were checked during palpitation, Valsalva maneuver, perinometry, and ultrasound and electromyographic investigation. Rankin
Scale, Mini Mental State Examination, Visual Analog Scale, and Time Up and Go test were also used. Patients' QoL before and after the treatment, assessed during each exam, were the study inclusion criteria. The following questionnaires were used in the QoL assessment: King's Health Questionnaire (KHQ), Incontinence Impact Questionnaire (IIQ), Urogenital Distress Inventory (UDI), QoL Scale (QoLS), Incontinence QoL (IQoL) questionnaire, Bristol Female Lower Urinary Tract Symptoms (BFLUTS) questionnaire, Health-Related Quality of Life, ICI Modular Questionnaire (ICIQ, ICIQUISF; short form; ICIQ-LUTSqol; Lower Urinary Tract Symptoms QoL), Ditrovie Scale, Pelvic Floor Distress Inventory (PFDI), Pelvic Floor Impact Questionnaire (PFIQ), and Symptom Impact Index (SII). Psychometric qualities, 
Table I Summary of studies' assessment of pelvic floor muscle training on incontinence-specific quality of life of women with urinary incontinence

\begin{tabular}{|c|c|c|c|c|c|c|c|}
\hline Reference & $\begin{array}{l}\text { Participants/ } \\
\text { age (years) }\end{array}$ & Type of UI & $\begin{array}{l}\text { Treatments } \\
\text { compared }\end{array}$ & $\begin{array}{l}\text { Treatment } \\
\text { duration }\end{array}$ & Outcome measures & Main findings & $\begin{array}{l}\text { Jadad } \\
\text { scale }\end{array}$ \\
\hline $\begin{array}{l}\text { Aslan et } \mathrm{al}^{12} \\
2008\end{array}$ & $\begin{array}{l}50 \text { women; } \\
\text { aged } 74-85\end{array}$ & SUI, UUI, MUI & $\begin{array}{l}\text { Supervised PFMT+BT } \\
\text { (EG) vs CG (without } \\
\text { exercises) }\end{array}$ & 6-8 weeks & $\begin{array}{l}\text { QoL (KHQ), pad test, } \\
\text { urinary diary, Mini- } \\
\text { mental test, Rankin } \\
\text { scale }\end{array}$ & $\begin{array}{l}\text { Statistically significant } \\
\text { decrease was observed } \\
\text { in the treatment group } \\
\text { compared to the control } \\
\text { group }\end{array}$ & $2 / 0 / 1$ \\
\hline $\begin{array}{l}\text { Balmforth } \\
\text { et al }{ }^{13} 2006\end{array}$ & $\begin{array}{l}97 \text { women; } \\
\text { aged } 40-60\end{array}$ & SUI & Supervised PFMT & 14 weeks & $\begin{array}{l}\text { QoL (KHQ), pad } \\
\text { test, UD, maximum } \\
\text { Valsalva maneuver, } \\
\text { bladder neck rotational } \\
\text { mobility from rest to } \\
\text { maximum incursion and } \\
\text { maximum excursion }\end{array}$ & $\begin{array}{l}\text { A statistically and } \\
\text { clinically significant } \\
\text { reduction in urine loss } \\
\text { and improvement in } \\
\text { condition-specific quality } \\
\text { of life }\end{array}$ & $2 / 0 / 1$ \\
\hline Bø et al ${ }^{14} 2000$ & $\begin{array}{l}59 \text { women; } \\
\text { aged } 40-55\end{array}$ & SUI & $\begin{array}{l}\text { PFMT (EG) vs CG } \\
\text { (without exercises) }\end{array}$ & $\begin{array}{l}24 \text { weeks; } \\
\text { EG } 45 \text { min } \\
\text { I } \times \text { week }\end{array}$ & $\begin{array}{l}\text { QoL (QoLS-N, } \\
\text { BFLUTS), PFM strength }\end{array}$ & $\begin{array}{l}\text { PFMT had a statistically } \\
\text { significant impact on QoL } \\
\text { and PFM strength }\end{array}$ & $2 / 0 / 1$ \\
\hline $\begin{array}{l}\text { Borello-France } \\
\text { et al }{ }^{15} 2006\end{array}$ & $\begin{array}{l}44 \text { women; } \\
\text { aged } 40-55\end{array}$ & SUI & $\begin{array}{l}\text { PFMT in supine } \\
\text { position (EG) vs } \\
\text { PFMT in supine } \\
\text { and standing } \\
\text { position (CG) }\end{array}$ & $9-12$ weeks & $\begin{array}{l}\text { QoL (IIQ), pad test, } \\
\text { bladder diary, UD, PFM } \\
\text { strength }\end{array}$ & $\begin{array}{l}\text { PFMT significantly } \\
\text { increased all test } \\
\text { parameters; exercise } \\
\text { position did not } \\
\text { differentially affect } \\
\text { treatment outcomes }\end{array}$ & $2 / 0 / 1$ \\
\hline $\begin{array}{l}\text { Bradley et al } \\
2010\end{array}$ & $\begin{array}{l}344 \text { women; } \\
\text { aged } 40-60\end{array}$ & SUI, UUI, MUI & $\begin{array}{l}\text { Supervised PFMT vs } \\
\text { continence pessary } \\
\text { vs combined }\end{array}$ & 12 weeks & $\begin{array}{l}\text { QoL (UDI), pad test, } \\
\text { urinary diary (7 days), } \\
\text { QUID }\end{array}$ & $\begin{array}{l}\text { All 3-month Ul outcomes } \\
\text { significantly improved } \\
\text { UDI scores }\end{array}$ & $2 / 0 / 1$ \\
\hline $\begin{array}{l}\text { Carneiro et al }{ }^{17} \\
2010\end{array}$ & $\begin{array}{l}50 \text { women; } \\
\text { aged } 40-60\end{array}$ & SUI & $\begin{array}{l}\text { Supervised PFMT } \\
\text { (EG) vs CG (without } \\
\text { exercises) }\end{array}$ & $\begin{array}{l}\text { EG } 8 \text { weeks; } \\
20 \text { min } \\
2 \times \text { week }\end{array}$ & $\begin{array}{l}\text { QoL (KHQ), bladder } \\
\text { diary UD, palpation } \\
\text { bidigital, surface EMG } \\
\text { motor activity PF }\end{array}$ & $\begin{array}{l}\text { PMFT positively } \\
\text { influenced the QoL in the } \\
\text { EG group }\end{array}$ & $2 / 0 / 1$ \\
\hline $\begin{array}{l}\text { de Oliveira } \\
\text { et al }{ }^{18} 2009\end{array}$ & $\begin{array}{l}60 \text { women; } \\
\text { aged } 40-60\end{array}$ & SUI & $\begin{array}{l}\text { Group PFMT (EG) } \\
\text { vs supervised PFMT } \\
\text { (individual) }\end{array}$ & $\begin{array}{l}\text { I } 2 \text { weeks; } \\
2 \times \text { week }\end{array}$ & $\begin{array}{l}\text { QoL (KHQ), pad test, } \\
\text { urinary diary (7-days) }\end{array}$ & $\begin{array}{l}\text { There were statistically } \\
\text { significant improvements } \\
\text { in both muscle strength } \\
\text { and quality of life. } \\
\text { When the groups were } \\
\text { compared, there were no } \\
\text { differences in the results } \\
\text { between them }\end{array}$ & $2 / 1 / 1$ \\
\hline $\begin{array}{l}\text { Demain et al }{ }^{19} \\
2001\end{array}$ & $\begin{array}{l}44 \text { women; } \\
\text { aged } 60\end{array}$ & SUI, UUI & $\begin{array}{l}\text { Supervised PFMT } \\
\text { EG (group sessions) } \\
\text { vs CG (individual } \\
\text { training) }\end{array}$ & $\begin{array}{l}\text { I2-I4 } \\
\text { weeks; EG } \\
3 \times \text { weeks vs } \\
\text { CG IXweek }\end{array}$ & $\begin{array}{l}\text { QoL (IIQ), pad test; } \\
\text { urinary diary (7 days), } \\
\text { incontinence impact } \\
\text { Questionnaire, } \\
\text { symptom severity } \\
\text { index, VAS }\end{array}$ & $\begin{array}{l}\text { EG results reached } \\
\text { statistical significance }\end{array}$ & $2 / 0 / 1$ \\
\hline $\begin{array}{l}\text { Dugan et } a^{20} \\
2013\end{array}$ & $\begin{array}{l}43 \text { women; } \\
\text { aged }>65\end{array}$ & $\mathrm{UI}$ & $\begin{array}{l}\text { Supervised PFMT } \\
\text { (EG) vs CG (only } \\
\text { education) }\end{array}$ & 6 weeks & $\begin{array}{l}\text { QoL (IIQ-7, UDI-6), } \\
\text { self-reported bladder } \\
\text { function, TUG }\end{array}$ & $\begin{array}{l}\text { A group pelvic floor } \\
\text { fitness program } \\
\text { combined with pelvic } \\
\text { health education } \\
\text { improved the QoL of } \\
\text { older women }\end{array}$ & $2 / 0 / 1$ \\
\hline Fan et $\mathrm{al}^{21} 2013$ & $\begin{array}{l}372 \text { women; } \\
\text { aged } 42-62\end{array}$ & SUI, UUI, MUI & Supervised PFMT & $\begin{array}{l}36-42 \\
\text { weeks }\end{array}$ & $\begin{array}{l}\text { QoL (UDI-6, IIQ-7), } \\
\text { UD }\end{array}$ & $\begin{array}{l}\text { PFMT appears to } \\
\text { be an effective first- } \\
\text { line intervention for } \\
\text { improving US and QoL } \\
\text { of women presenting } \\
\text { with UI }\end{array}$ & $2 / 0 / 1$ \\
\hline
\end{tabular}


Table I (Continued)

\begin{tabular}{|c|c|c|c|c|c|c|c|}
\hline Reference & $\begin{array}{l}\text { Participants/ } \\
\text { age (years) }\end{array}$ & Type of UI & $\begin{array}{l}\text { Treatments } \\
\text { compared }\end{array}$ & $\begin{array}{l}\text { Treatment } \\
\text { duration }\end{array}$ & Outcome measures & Main findings & $\begin{array}{l}\text { Jadad } \\
\text { scale }\end{array}$ \\
\hline $\begin{array}{l}\text { Felicíssimo } \\
\text { et } \mathrm{al}^{22} 2010\end{array}$ & $\begin{array}{l}62 \text { women; } \\
\text { aged } 45-60\end{array}$ & SUI & $\begin{array}{l}\text { Supervised } \\
\text { PFMT (EG) vs CG } \\
\text { (training at home) }\end{array}$ & $\begin{array}{l}8 \text { weeks; } \\
\text { EG } 2 \times \text { week, } \\
50 \text { min; CG } \\
90-180 \\
\text { contr/day }\end{array}$ & $\begin{array}{l}\text { QoL (ICIQ-SFF), } \\
\text { urogynecological } \\
\text { history, urodynamic } \\
\text { study }\end{array}$ & $\begin{array}{l}\text { Both intensive, } \\
\text { supervised PFMT and } \\
\text { unsupervised PFMT are } \\
\text { effective to treat female } \\
\text { SUI if training is provided }\end{array}$ & $2 / 0 / 1$ \\
\hline $\begin{array}{l}\text { Ferreira and } \\
\text { Santos }^{23} 2012\end{array}$ & $\begin{array}{l}34 \text { women; } \\
\text { aged } 50\end{array}$ & SUI & $\begin{array}{l}\text { Supervised } \\
\text { PFMT (EG) vs CG } \\
\text { (training at home) }\end{array}$ & $\begin{array}{l}24 \text { weeks; } \\
\text { EG } 45 \text { min } \\
\text { I×week }\end{array}$ & $\begin{array}{l}\text { QoL (Ditrovie scale), } \\
\text { number of incontinence } \\
\text { episodes }\end{array}$ & $\begin{array}{l}\text { IQoL improved in both } \\
\text { groups but EG }>C G\end{array}$ & $2 / 2 / 1$ \\
\hline $\begin{array}{l}\text { Fitz et al }{ }^{24} \\
2012\end{array}$ & $\begin{array}{l}36 \text { women; } \\
\text { aged } 46-64\end{array}$ & SUI & Supervised PFMT & $\begin{array}{l}12 \text { weeks; } \\
3 \times \text { week }\end{array}$ & $\begin{array}{l}\text { QoL (KHQ), urinary } \\
\text { diary (7-days), PFM } \\
\text { function }\end{array}$ & $\begin{array}{l}\text { PFM training resulted in } \\
\text { significant improvement } \\
\text { in the QoL of women } \\
\text { with UI }\end{array}$ & $2 / 0 / 1$ \\
\hline $\begin{array}{l}\text { Hung et } \mathrm{al}^{25} \\
2010\end{array}$ & $\begin{array}{l}70 \text { women; } \\
\text { aged } 50-60\end{array}$ & SUI, MUI & $\begin{array}{l}\text { Supervised } \\
\text { PFMT (EG) vs CG } \\
\text { (training at home) }\end{array}$ & 16 weeks & $\begin{array}{l}\text { QoL (SII), number of } \\
\text { incontinence episodes, } \\
\text { PFM function, } 20 \text { min } \\
\text { pad test; 3-day voiding } \\
\text { diary }\end{array}$ & $\begin{array}{l}\text { More aspects of quality of } \\
\text { life improved significantly } \\
\text { in the training group than } \\
\text { in the control group }\end{array}$ & $2 / 0 / 1$ \\
\hline $\begin{array}{l}\text { Jahromi et } \mathrm{al}^{26} \\
2015\end{array}$ & $\begin{array}{l}50 \text { women, } \\
\text { aged } 60-74\end{array}$ & SUI & $\begin{array}{l}\text { PFMT (EG) vs CG } \\
\text { (without exercises) }\end{array}$ & $\begin{array}{l}8 \text { weeks; } \\
\text { I×week }\end{array}$ & $\begin{array}{l}\text { QoL (ICIQ), number of } \\
\text { incontinence episodes, } \\
\text { QUID, self-esteem } \\
\text { questionnaires }\end{array}$ & $\begin{array}{l}\text { ICIQ score has a } \\
\text { significant difference } \\
\text { between the } 2 \text { groups }\end{array}$ & $2 / 0 / 1$ \\
\hline $\begin{array}{l}\text { Kaya et } \mathrm{al}^{27} \\
2015\end{array}$ & $\begin{array}{l}\text { I08 women; } \\
\text { aged } 40-60\end{array}$ & SUI, UUI, MUI & $\begin{array}{l}\mathrm{PFMT}+\mathrm{BT}(\mathrm{EG}) \text { vs } \\
\mathrm{BT}(\mathrm{CG})\end{array}$ & 6 weeks & $\begin{array}{l}\text { QoL (IIQ-7, UDI-6), } \\
\text { number of incontinence } \\
\text { episodes, PFM strength } \\
\text { and endurance }\end{array}$ & $\begin{array}{l}\text { High-intensity PFMT } \\
\text { combined with BT is } \\
\text { more effective than BT } \\
\text { alone in the short term } \\
\text { for treating UI or SUI }\end{array}$ & $2 / 0 / 1$ \\
\hline $\begin{array}{l}\text { Kenton K } \\
\text { et } \mathrm{al}^{28} 20 \mathrm{I} 2\end{array}$ & $\begin{array}{l}446 \text { women; } \\
\text { aged } 40-60\end{array}$ & SUI & $\begin{array}{l}\text { Pessary therapy } \\
\text { vs PFMT vs CG } \\
\text { (combination } \\
\text { therapy) }\end{array}$ & $\begin{array}{l}\text { I } 2 \text { weeks; } \\
2 \times 1 \text { week }\end{array}$ & $\begin{array}{l}\text { QoL (PFDI, PFIQ), } \\
\text { number of incontinence } \\
\text { episodes, urinary diary } \\
\text { (7 days), QUID }\end{array}$ & $\begin{array}{l}\text { There was no difference } \\
\text { in pelvic floor symptom } \\
\text { bother and HRQoL } \\
\text { between the pessary and } \\
\text { behavioral therapy arms } \\
\text { in women undergoing } \\
\text { conservative treatment } \\
\text { for SUI }\end{array}$ & $2 / 1 / 1$ \\
\hline $\begin{array}{l}\text { Konstantinidou } \\
\text { et } \mathrm{al}^{29} 2013\end{array}$ & $\begin{array}{l}46 \text { women; } \\
\text { aged } 40-60\end{array}$ & SUI & $\begin{array}{l}\text { EG (TrA+PFMT) vs } \\
\text { CG (PFMT) }\end{array}$ & 12 weeks & $\begin{array}{l}\text { QoL (KHQ), 7-day } \\
\text { bladder diary, vaginal } \\
\text { assessment of the } \\
\text { PFM strength using } \\
\text { the Oxford grading, } \\
\text { 24-hour pad test }\end{array}$ & $\begin{array}{l}\text { TrA+PFMT gave no } \\
\text { additional benefit } \\
\text { compared to PFMT in } \\
\text { improvement of research } \\
\text { results, in particular, } \\
\text { QoL. }\end{array}$ & $2 / 0 / 1$ \\
\hline $\begin{array}{l}\text { Nascimento- } \\
\text { Correia et } \mathrm{al}^{30} \\
2012\end{array}$ & $\begin{array}{l}30 \text { women; } \\
\text { aged } 50-69\end{array}$ & UI & $\begin{array}{l}\text { PFMT (EG) vs CG } \\
\text { (without exercise) }\end{array}$ & $\begin{array}{l}\text { I2 weeks; } \\
\text { I-hour } \\
\text { sessions }\end{array}$ & $\begin{array}{l}\text { QoL (KHQ), number } \\
\text { of incontinence } \\
\text { episodes, PFM function }\end{array}$ & $\begin{array}{l}\text { The protocol to } \\
\text { strengthen the PFM used } \\
\text { by the EG was effective } \\
\text { to improve the UI, QOL, } \\
\text { function and pressure of } \\
\text { PFM contraction }\end{array}$ & $2 / 0 / 1$ \\
\hline $\begin{array}{l}\text { Pereira et } \mathrm{al}^{31} \\
2012\end{array}$ & $\begin{array}{l}41 \text { women; } \\
\text { aged } 50-60\end{array}$ & SUI & $\begin{array}{l}\text { PFMT EG (group } \\
\text { sessions) vs VG } \\
\text { (vaginal cones) vs CG } \\
\text { (without exercises) }\end{array}$ & $\begin{array}{l}6 \text { weeks; } \\
\text { EG } 40 \text { min } \\
2 \times \text { week; } \\
\text { VG } 40 \text { min } \\
2 \times \text { week }\end{array}$ & $\begin{array}{l}\text { QoL (KHQ), pressure } \\
\text { perineometry, PFM } \\
\text { strength by digital } \\
\text { palpation }\end{array}$ & $\begin{array}{l}\text { PFMT and VG both } \\
\text { treatments are effective } \\
\text { in UI }\end{array}$ & $2 / 0 / 1$ \\
\hline $\begin{array}{l}\text { Ptak et } \mathrm{al}^{32} \\
2017\end{array}$ & $\begin{array}{l}\text { I } 40 \text { women; } \\
\text { aged }>50\end{array}$ & SUI & $\begin{array}{l}\text { EG (TrA+PFMT) vs } \\
\text { CG (PFMT) }\end{array}$ & $\begin{array}{l}12 \text { weeks; } \\
3 \times \text { week }\end{array}$ & $\begin{array}{l}\text { QoL (ICIQ-LUTSqol), } \\
\text { Gaudenz questionnaire, } \\
\text { physical examination, } \\
\text { and urodynamic testing }\end{array}$ & $\begin{array}{l}\text { Both groups showed a } \\
\text { significant improvement } \\
\text { in most QoL domains } \\
\text { measured with ICIQ } \\
\text { LUTS QoL }\end{array}$ & $2 / 1 / 0$ \\
\hline
\end{tabular}


Table I (Continued)

\begin{tabular}{|c|c|c|c|c|c|c|c|}
\hline Reference & $\begin{array}{l}\text { Participants/ } \\
\text { age (years) }\end{array}$ & Type of UI & $\begin{array}{l}\text { Treatments } \\
\text { compared }\end{array}$ & $\begin{array}{l}\text { Treatment } \\
\text { duration }\end{array}$ & Outcome measures & Main findings & $\begin{array}{l}\text { Jadad } \\
\text { scale }\end{array}$ \\
\hline $\begin{array}{l}\text { Sar and } \\
\text { Khorshid }^{33} \\
2009\end{array}$ & $\begin{array}{l}41 \text { women; } \\
\text { aged } 40-55\end{array}$ & SUI, MUI & $\begin{array}{l}\text { PFMT (EG) vs CG } \\
\text { (without training) }\end{array}$ & $\begin{array}{l}8 \text { weeks; } \\
3 \text { sets daily }\end{array}$ & $\begin{array}{l}\text { QoL (I - QoL), number } \\
\text { of incontinence } \\
\text { episodes, pad test, PFM } \\
\text { strength }\end{array}$ & $\begin{array}{l}\text { PFMT significantly } \\
\text { increased PFM strength, } \\
\text { QOL and reduced the } \\
\text { frequency of Ul episodes }\end{array}$ & $2 / 0 / 1$ \\
\hline $\begin{array}{l}\text { Sherburn } \\
\text { et } \mathrm{al}^{34} 20 \mathrm{ll}\end{array}$ & $\begin{array}{l}83 \text { women; } \\
\text { aged }>65\end{array}$ & SUI & $\begin{array}{l}\text { Supervised PFMT } \\
\text { vs PFMT (training } \\
\text { at home) vs CG } \\
\text { (bladder training) }\end{array}$ & 20 weeks & $\begin{array}{l}\text { QoL (ICIQ-SF), Urine } \\
\text { loss on a stress test; } \\
\text { UD, VAS, urinary diary } \\
\text { (7 days), 5-point global } \\
\text { rating of change; TUG } \\
\text { test }\end{array}$ & $\begin{array}{l}\text { High intensity PFMT is } \\
\text { effective in managing } \\
\text { stress urinary } \\
\text { incontinence and is more } \\
\text { effective than BT in } \\
\text { healthy older women }\end{array}$ & $2 / 2 / 1$ \\
\hline $\begin{array}{l}\text { Zanetti et al }{ }^{35} \\
2007\end{array}$ & $\begin{array}{l}44 \text { women; } \\
\text { aged 53-57 }\end{array}$ & SUI & $\begin{array}{l}\text { Supervised PFMT } \\
\text { (EG) vs CG (training } \\
\text { at home) }\end{array}$ & $\begin{array}{l}\text { I2 weeks; } \\
\text { EG } 2 \times \text { week, } \\
45 \text { min; CG } \\
\text { I×4week }\end{array}$ & $\begin{array}{l}\text { QoL (IQoL), pad test, } \\
\text { urinary diary (7-days), } \\
\text { PFM strength }\end{array}$ & $\begin{array}{l}\text { There was a significant } \\
\text { increase in the QoL of } \\
\text { patients after treatment. }\end{array}$ & $2 / 0 / 1$ \\
\hline
\end{tabular}

Abbreviations: BFLUTS, Bristol Female Lower Urinary Tract Symptoms Questionnaire; BT, bladder training; Contr, contractions; CG, control group; EG, experimental group; EMG, electromyography; HRQoL, Health-Related Quality of Life; ICIQ, International Consultation on Incontinence Questionnaire; ICIQ LUTSqol, International Consultation Incontinence Questionnaire Lower Urinary Tract Symptoms quality of life; ICIQ-SF, International Consultation on Incontinence Questionnaire-Short Form; IIQ, Incontinence Impact Questionnaire; IQoL, Incontinence Quality of Life Questionnaire; KHQ, King's Health Questionnaire; MUI, mixed urinary incontinence; PFDI, pelvic floor distress inventory; PFDI, Ditrovie Scale, Pelvic Floor Distress Inventory; PFIQ, pelvic floor impact questionnaire; PFMT, pelvic floor muscle training; PFM, pelvic floor muscle; QoL, quality of Life; QoLS-N, Norwegian version of the Quality of Life Scale; QUID, Questionnaire for Urinary Incontinence Diagnosis; SII, Symptom Impact Index; SUI, stress urinary incontinence; TrA, abdominal muscle training; TUG, time up and go test; UD, ultrasound; UDI, urogenital distress inventory; UI, urinary incontinence; UIQ, urinary impact questionnaire; US, urinary symptoms; UUI, urge urinary incontinence; VAS, visual analog scale; VG, vaginal cones.

accuracy, and credibility of all the questionnaires were confirmed clinically.

The most commonly used questionnaire was the KHQ; it was mentioned in 8 out of 24 studies. ${ }^{12,13,17,18,24,28-31}$ KHQ is a patient self-administered self-report and has 3 parts consisting of 21 items. Part 1 contains general health perception and incontinence impact ( 1 item each). Part 2 contains role limitations, physical limitations, social limitations (2 items each), personal relationships, emotions ( 3 items each) and sleep/energy ( 2 items), as well as severity measures (4 items). Part 3 is considered as a single item and contains 10 responses in relation to frequency, nocturia, urgency, urge, stress, intercourse incontinence, nocturnal enuresis, infections, pain, and difficulty in voiding. ${ }^{36} \mathrm{KHQ}$ is a tool recommended by the European Clinical Practice Guidelines. ${ }^{37}$

In 5 out of 24 studies, the QoL was evaluated by the use of IIQ. ${ }^{15,19,20,21,27}$ This questionnaire was used in 2 versions: the full version - IIQ and the abbreviated one IIQ-7. In 3 out of 24 studies, the full version of the questionnaire was used, ${ }^{15,19,20}$ while in 2 out of 24 tests, a short form - IIQ-7 was used. ${ }^{21,27}$ The IIQ was designed by Shumaker et $\mathrm{al}^{38}$ to assess the impact of UI on activities and emotions in women. Thirty self-administered questions cover 4 domains: physical activity, social relationships, traveling, and emotional health.

In 4 out of 24 studies, the ICIQ was applied. ${ }^{22,26,32,34}$ The ICIQ-SF consists of 3 items (Frequency, Amount, and Impact), and a group of 8 questions related to the type of UI, which are not part of the questionnaire score. The purpose of the 8 questions is to describe a given type of urinary incontinency. The total score is the sum of the first 3 items and it ranges from 0 to 21 points. A score of 21 - the worst possible QoL, and 0 - the best possible QoL. ${ }^{39}$ ICIQ LUTS QoL is based on KHQ. ICIQ LUTS measures the impact of UI on physical and mental activities, changes in interpersonal relations, and in everyday life. The global score can range between 19 and 79 points - the higher the score, the worse the QoL. The conversion of points of ICIQ LUTS QoL is carried out on the basis of the instructions by Hebbar based on KHQ. ${ }^{36,40}$

In 2 out of 24 tests, the IQoL questionnaire was implemented. ${ }^{22,26,28}$ The IQoL questionnaire is composed of 20 questions evaluating limitations on behavior, the psychosocial impact, and the social embarrassment that UI causes. $^{41}$

The scales, which have only been used in 1 study are as follows, The Norwegian version of the QoL Scale (QoLS-N), ${ }^{14}$ The BFLUTS questionnaire, ${ }^{14}$ PFDI, ${ }^{28}$ PFIQ, ${ }^{28}$ SII, ${ }^{25}$ and Ditrovie scale. ${ }^{23}$ The QoLS-N is adapted and modified for use in the chronic illness population, by Burckhardt et $\mathrm{al}^{4}{ }^{42}$ this scale applies to general health and QoL. The Norwegian version uses a 7-point satisfaction scale. ${ }^{43}$ The BFLUTS questionnaire was designed to assess a wide range of symptoms, including incontinence, and the impact on sexual function and QoL. ${ }^{44}$ The PFDI comprises a urinary scale (UDI; 
score range 0-300), including 3 subscales (Stress, Irritative and Obstructive; score range 0-100), prolapse (POPDI; score range 0-300), and colorectal (CRADI; score range 0-400) scales. Higher PFDI scale and subscale scores reflect increasing symptoms. The PFIQ includes 3 scales, urinary (UIQ), prolapse (POPIQ) and colorectal (CRAIQ) ones, each with score range of $0-300$. Higher PFIQ scores - greater daily impact on pelvic symptoms. ${ }^{45}$ The Ditrovie scale consists of 10 items: activity (4 items), emotional impact ( 2 items), selfimage (2 items), sleep (1 item), and general welfare (1 item). The higher the score, the worse the QoL. ${ }^{46}$ The SII measures women's views on stress incontinence severity, as well as the impact or discomfort caused by the symptoms. ${ }^{47}$

\section{Therapy comparison and treatment duration}

The studies compared supervised and unsupervised PFMT, group and individual training, PFMT and bladder training (also with the use of vaginal cones), as well as training groups and a control group (no therapy). Treatment duration was between 6 and 42 weeks; a majority of the therapy sessions were held 1-3 times per week, and lasted 45 minutes each.

\section{Pelvic floor muscles training and types of UI}

The evaluation of the impact of PFMT on the QoL of women with incontinence was carried out in all of the presented studies. In 16 studies, the study population concerned women with SUI. Moreover, in studies, the authors compared the effects of pelvic floor muscle exercises on incontinence depending on the type of urinary incontinence. ${ }^{16,21,27}$ In addition, each study showed that the statistically highest improvement in QoL occurred in SUI, then a lower result was achieved in UUI, and no significant differences were found in the impact of PFMT on MUI. In the case of 2 studies, the type of UI in the study population was not reported. ${ }^{20,30}$

\section{Compared treatment methods}

A PFM training group and a control group (no training) were compared in 5 out of the 24 studies which qualified for the analysis (223 patients). ${ }^{14,17,26,30,33}$ In all referenced studies, statistically significant improvement in the QoL in the PFMT group was observed compared with the control group.

In 5 out of the 24 studies (a total of 253 women) qualified for the analysis, a PFM training group was divided into a supervised PFMT group and a control (home-exercise) group. Fan et $\mathrm{al}^{21}$ study results showed a significant improvement of the UDI total result $(p<0.01)$ and the IIQ $(p<0.01)$ in the experimental group compared with the control group after a 6-week training program. Whereas Ferreira et a ${ }^{23}$ showed that 6 months following the treatment, the QoL improved in both groups. However, the outcome was better in women in the supervised PFMT group than in women who exercised at home $(p=0.04)$. Similar study results were obtained by Sherburn et $\mathrm{al}^{34}$ and Jahromi et al. ${ }^{26}$ Felicissimo et $\mathrm{al}^{22}$ showed there were no differences between the supervised group and the home-exercise group, with regard to the QoL $(p=0.76)$. The study authors concluded that both supervised PFMT and unsupervised PFMT are effective in UI treatment, if a proper training session is provided.

The effectiveness of group and individual training was compared in 2 out of the 24 selected studies (104 patients). ${ }^{18,19}$ No significant differences were found between both studies in terms of group and individual training effectiveness.

In 1 out of the 24 selected studies (44 women), PFMT in supine position (KG) was compared with PFMT in supine and standing position (CG). The study results have shown the effectiveness of PFMT, but various training positions had no impact on its effectiveness. ${ }^{15}$

In 8 out of the 24 selected studies, the effectiveness of PFMT was compared with other methods. In studies conducted by Pereira et al, ${ }^{31}$ a group of 45 women was divided into 3 distinct groups - a vaginal cone group (VG), a PFMT group, and a control group (no therapy). After 6 weeks of therapy, a significant improvement $(p<0.01)$ in terms of QoL was observed in both the VG group and the PFMT group, compared with the control group. The following 2 studies (186 women) compared abdominal muscle training ( $\operatorname{TrA}$ ) to PFMT monotherapy. Konstantinidou et $\mathrm{al}^{29}$ found that improvement in QoL was observed in both groups, and no significant differences were found between them. Whereas, Ptak et $\mathrm{al}^{32}$ observed better results in the combined therapy group (TrA+PFMT).

In 3 out of the 24 selected studies, bladder training (BT) was compared with PFMT. A total of 238 women participated in the study. Sherburn et $\mathrm{al}^{34}$ showed an improvement in QoL in both groups $(p=0.01)$, without any significant differences between the groups. The next study compared a combined PFMT/BT with a BT monotherapy. ${ }^{27}$ After treatment, all of the studied women had an improvement in QoL (IIQ-7 $p=0.01$; UDI-6 $p<0.01$ ). The following study (790 patients) compared the effectiveness of combined PFMT/BT (the study group) to no treatment (the control group). After therapy, there was a statistically significant improvement in QoL in the study group, compared with the control group $(p=0.05))^{12}$ 
In 2 out of the 24 selected studies (790 women), QoL was evaluated after PFMT, pessary therapy, and combined therapy (PFMT+pessary therapy). ${ }^{16,28}$ After a 3-month treatment, a statistically significant improvement in QoL $(p<0.01)$ for all women was observed in both studies, and no significant differences were observed between the groups.

In 3 out of the 24 selected studies ( 505 women), patients received PFMT monotherapy and there was no control group. ${ }^{13,21,24}$ Each study showed a statistically significant improvement in QoL after PFMT.

\section{Limitations}

The limitation of this analysis is primarily the methodical variability in the analyzed studies. The search criteria should be narrowed further, taking into account the methodological homogeneity of the conducted therapy.

\section{Conclusion}

The results of this literature review demonstrate that PFMT is an effective treatment for UI in women. PFMT appears to be an effective non-surgical intervention, particularly for women with SUI. It could also be recommended as the first-line conservative treatment for elderly women. PFMT significantly improves the QoL of women with UI, which is an important determinant of their physical, mental, and social functioning. The duration of PFMT should not be shorter than 6 weeks. It is advised to provide supervised PFMT. PFMT can be used either as a monotherapy or as a combined therapy for the treatment of UI in women. The KHQ is useful for following up on the QoL of women with UI in many different clinical settings and in controlled clinical trials.

\section{Author contributions}

All authors contributed toward data analysis, drafting and critically revising the paper, gave final approval of the version to be published, and agree to be accountable for all aspects of the work.

\section{Disclosure}

The authors report no conflicts of interest in this work.

\section{References}

1. The Global Forum on Incontinence. About incontinence. Available from: http://www.gfiforum.com/incontinence. Accessed January 15, 2018.

2. Abrams P, Cardozo L, Wagg A, Wein A. Incontinence, 6th edition 2017. 6th International Consultation on Incontinence, Tokyo, September, s. 22, 87-88.

3. Edwall L, Carlstrom K, Jonasson AF. Different estrogen sensitivity of urogenital tissue from women with and without stress urinary incontinence. Neurourol Urodyn. 2009;28:516-520.
4. Melville JL, Fan MY, Rau H, Nygaard IE, Katon WJ. Major depression and urinary incontinence in women: temporal associations in an epidemiologic sample. Am J Obstet Gynecol. 2009;201(5):490-497.

5. van der Vaart CH, Roovers JP, de Leeuw JR, Heintz AP. Association between urogenital symptoms and depression in community-dwelling women aged 20 to 70 years. Urology. 2007;69(4):691-696.

6. Felde G, Bjelland I, Hunskaar S. Anxiety and depression associated with incontinence in middle-aged women: a large Norwegian crosssectional study. Int Urogynecol J. 2012;23(3):299-306.

7. Tettamanti G, Altman D, Iliadou AN, Bellocco R, Pedersen NL. Depression, neuroticism, and urinary incontinence in premenopausal women: a nationwide twin study. Twin Res Hum Genet. 2013;16(5):977-984.

8. Farage MA, Miller KW, Berardesca E, Maibach HI. Psychosocial and societal burden of incontinence in the aged population: a review. Arch Gynecol Obstet. 2008;277(4):285-290.

9. Kegel A, Powell T. The physiologic treatment of urinary stress incontinence. J Urol. 1950;63:808-814.

10. Liberati A, Altman DG, Tetzlaff J, et al. The PRISMA statement for reporting systematic reviews and meta-analyses of studies that evaluate healthcare interventions: explanation and elaboration. $B M J$. 2009;339:b2700.

11. Jadad AR, Moore RA, Dawn C, et al. Assessing the quality of reports of randomized clinical trials: is blinding necessary? Control Clin Trials. 1996;17:1-12.

12. Aslan E, Komurcu N, Beji NK, Yalcin O. Bladder training and Kegel exercises for women with urinary complaints living in a rest home. Gerontology. 2008;54(4):224-231.

13. Balmforth JR, Mantle J, Bidmead J, Cardozo L. A prospective observational trial of pelvic floor muscle training for female stress urinary incontinence. BJU Int. 2006;98(4):811-817.

14. Bø K, Talseth T, Vinsnes A. Randomized controlled trial on the effect of pelvic floor muscle training on quality of life and sexual problems in genuine stress incontinent women. Acta Obstet Gynecol Scand. 2000; 79(7):598-603.

15. Borello-France DF, Zyczynski HM, Downey PA, Rause CR, Wister JA. Effect of pelvic-floor muscle exercise position on continence and quality-of-life outcomes in women with stress urinary incontinence. Phys Ther. 2006;86(7):974-986.

16. Bradley CS, Rahn DD, Nygaard IE, et al. The questionnaire for urinary incontinence diagnosis (QUID): validity and responsiveness to change in women undergoing non-surgical therapies for treatment of stress predominant urinary incontinence. Neurourol Urodyn. 2010; 29(5):727-734.

17. Carneiro EF, Araujo Ndos S, Beuttenmüll L, et al. The anatomicalfunctional characteristics of the pelvic floor and quality of life of women with stress urinary incontinence subjected to perineal exercises. Actas Urol Esp. 2010;34(9):788-793.

18. de Oliveira Camargo F, Rodrigues AM, et al. Pelvic floor muscle training in female stress urinary incontinence: comparison between group training and individual treatment using PERFECT assessment scheme. Int Urogynecol J Pelvic Floor Dysfunct. 2009;20(12):1455-1462.

19. Demain S, Smith JD, Hiller L, Dziedzic K. Comparison of group and individual physiotherapy for female urinary incontinence in primary care: pilot study. Physiotherapy. 2001;87(5):235-242.

20. Dugan SA, Lavender MD, Hebert-Beirne J, Brubaker L. A pelvic floor fitness program for older women with urinary symptoms: a feasibility study. PMR. 2013;5(8):672-676.

21. Fan HL, Chan SS, Law TS, Cheung RY, Chung TK. Pelvic floor muscle training improves quality of life of women with urinary incontinence: a prospective study. Aust N ZJ Obstet Gynaecol. 2013;53(3):298-304.

22. Felicíssimo MF, Carneiro MM, Saleme CS, Pinto RZ, da Fonseca AM, da Silva-Filho AL. Intensive supervised versus unsupervised pelvic floor muscle training for the treatment of stress urinary incontinence: a randomized comparative trial. Int Urogynecol J. 2010;21(7):835-840.

23. Ferreira M, Santos PC. Impact of exercise programs in woman's quality of life with stress urinary incontinence. Rev Port Saude Publica. 2012; 3(1):3-10. 
24. Fitz FF, Costa TF, Yamamoto DM, et al. [Impact of pelvic floor muscle training on the quality of life in women with urinary incontinence]. Rev Assoc Med Bras (1992). 2012;58(2):155-159. Portuguese.

25. Hung HC, Hsiao SM, Chih SY, Lin HH, Tsauo JY. An alternative intervention for urinary incontinence: retraining diaphragmatic, deep abdominal and pelvic floor muscle coordinated function. Man Ther. 2010;15(3):273-279.

26. Jahromi MK, Talebizadeh M, Mirzaei M. The effect of pelvic muscle exercises on urinary incontinency and self-esteem of elderly females with stress urinary incontinency. Glob J Health Sci. 2015;2(7):71-79.

27. Kaya S, Akbayrak T, Gursen C. Short-term effect of adding pelvic floor muscle training to bladder training for female urinary incontinence: a randomized controlled trial. Int Urogynecol J. 2015;26(2):285-293.

28. Kenton K, Barber M, Wang L, et al. Pelvic floor symptoms improve similarly after pessary and behavioral treatment for stress incontinence. Female Pelvic Med Reconstr Surg. 2012;18(2):118-121.

29. Konstantinidou E, Kalaitzi M, Mytilekas KV, Ioannides EI, Hatzichristou D, Apostolidis A. Does the type of physiotherapy affect the quality of life and clinical outcomes in female urinary incontinence? A comparative study of two physiotherapy schemes. Eur Urol Suppl. 2013;12(1):e733.

30. Nascimento-Correia G, Santos-Pereira V, Tahara N, Driusso P. [Effects of pelvic floor muscle training on quality of life of a group of women with urinary incontinence: randomized controlled trial]. Actas Urológicas Españolas (English Edition). 2012;36(4):216-221. Spanish.

31. Pereira VS, de Melo MV, Correia GN, Driusso P. Vaginal cone for postmenopausal women with stress urinary incontinence: randomized, controlled trial. Climacteric. 2012;15(1):45-51.

32. Ptak M, Brodowska A, Ciećwież S, Rotter I. Quality of life in women with stage 1 stress urinary incontinence after application of conservative treatment-A randomized trial. Int J Environ Res Public Health. 2017;14(6):577.

33. Sar D, Khorshid L. The effects of pelvic floor muscle training on stress and mixed urinary incontinence and quality of life. $J$ Wound Ostomy Continence Nurs. 2009;36(4):429-435.

34. Sherburn M, Bird M, Carey M, Bø K, Galea MP. Incontinence improves in older women after intensive pelvic floor muscle training: an assessor-blinded randomized controlled trial. Neurourol Urodyn. 2011;30(3):317-324.

35. Zanetti MR, Castro Rde A, Rotta AL, Santos PD, Sartori M, Girão MJ. Impact of supervised physiotherapeutic pelvic floor exercises for treating female stress urinary incontinence. Sao Paulo Med J. 2007; 125(5):265-269
36. Hebbar S, Pandey H, Chawla A. Understanding Kings Health Questionnaire (KHQ) in assessment of female urinary incontinence. Int J Res Med Sci. 2015;3:531-538.

37. Viktrup L, Summers KH, Dennett SL. Clinical practice guidelines for the initial management of urinary incontinence in women: a Europeanfocused review. BJU Int. 2004;94(1):14-22.

38. Shumaker SA, Wyman JF, Uebersax JS, McClish D, Fantl JA, for the Continence Program in Women (CPW) Research Group. Health-related quality of life measures for women with urinary incontinence: the incontinence impact questionnaire and the urogenital distress inventory. Qual Life Res. 1994;3:291-306.

39. Tamanini JT, Dambros M, D’Ancona CA, Palma PC, RodriguesNetto N Jr Responsiveness to the Portuguese version of the International Consultation on Incontinence Questionnaire-Short Form (ICIQ-SF) after urinary stress incontinence surgery. Int Braz J Urol. 2005;31:482-489.

40. Kelleher CJ, Cardozo LD, Khullar V, Salvatore S. A new questionnaire to assess the quality of life of urinary incontinent women. Br J Obstet Gyneacol. 1997;140:1374-1379.

41. Patrick DL, Martin ML, Bushnell DM, Yalcin I, Wagner TH, Buesching DP. Quality of life of women with urinary incontinence: further development of the incontinence quality of life instrument (I-QOL). Urology. 1999;53(1):71-76.

42. Burckhardt C, Woods S, Schultz A, Ziebarth D. Quality of life in adults with chronic illness: a psychometric study. Res Nurs Health. 1989;12: 347-354.

43. Wahl A, Burckhardt C, Wiklund I, Hanestad V. The Norwegian version of the quality of life scale (QoLS-N). Scand J Caring Sci. 1998;12: 215-222.

44. Jackson S, Donovan J, Brookes S, Eckford S, Swithinbank L, Abrams P. The Bristol female lower urinary tract symptoms questionnaire: development and psychometric testing. Br J Urol. 1996;77:805-812.

45. Barber MD, Spino C, Janz NK, et al. The minimum important differences for the urinary scales of the Pelvic Floor Distress Inventory and Pelvic Floor Impact Questionnaire. Am J Obstet Gynecol. 2009; 200:580.

46. Amarenco G, Marquis P, Leriche B, Richard F, Zerbib M, Jacquetin B. A specific scale for assessing the disruption of quality of life during voiding disorders: scale Ditrovie. Ann Réadapt Méd Phys. 1997;40: 21-26.

47. Black N, Griffiths J, Pope C. Development of a symptom severity index and a symptom impact index for stress incontinence in women. Neurourol Urodyn. 1996;15(6):630-640.
Clinical Interventions in Aging

\section{Publish your work in this journal}

Clinical Interventions in Aging is an international, peer-reviewed journal focusing on evidence-based reports on the value or lack thereof of treatments intended to prevent or delay the onset of maladaptive correlates of aging in human beings. This journal is indexed on PubMed Central, MedLine,

\section{Dovepress}

CAS, Scopus and the Elsevier Bibliographic databases. The manuscript management system is completely online and includes a very quick and fair peer-review system, which is all easy to use. Visit http://www.dovepress. com/testimonials.php to read real quotes from published authors. 